\title{
Evaluation of Be and Li cross section data up to $200 \mathrm{MeV}$
}

\author{
N. Shigyo ${ }^{1, a}$, K. Ishibashi ${ }^{1}$, and S. Kunieda ${ }^{2}$ \\ 1 Department of Applied Quantum Physics and Nuclear Engineering, Kyushu University, Fukuoka 813-0395, Japan \\ 2 Japan Atomic Energy Agency, Tokai, 319-1195 Japan
}

\begin{abstract}
Nuclear cross section data on lithium and beryllium isotopes are evaluated in the incident energy up to $200 \mathrm{MeV}$ for proton and neutron incidence. For the first step for overall evaluation, parameters for phenomenological optical potential are obtained. Evaluated cross sections include neutron total cross section, total reaction cross section, angular differential elastic scattering cross section, nonelastic cross section.
\end{abstract}

\section{Introduction}

Both lithium and beryllium play an important role as a neutron converter for the boron neutron capture therapy (BNCT) using a proton accelerator. Beryllium is also one of essential materials as a target window and a reflector for a spallation neutron source. Evaluated nuclear data for these nuclei are contained in the energy region below $20 \mathrm{MeV}$ in the JENDL [1] at the moment and those in higher energy region are desired for such kinds of application. In this study, nuclear cross section data on $\mathrm{Li}$ and $\mathrm{Be}$ isotopes are evaluated in the incident energy up to $200 \mathrm{MeV}$ for proton and neutron incidence as the first step of overall evaluation.

The evaluated cross section data include neutron total cross section, total reaction cross section, angular differential elastic scattering cross section, nonelastic cross section and double differential secondary particle production cross section. The phenomenological optical model parameters are found to give good agreement with experimental data of total, elastic- and nonelastic-scattering cross sections. These evaluated cross sections are compared with available experimental values. The evaluated nuclear data will be merged into the JENDL High Energy File (JENDL-HE) [2] in the near future after evaluation of particle emission cross sections by the GNASH code [3].

\section{Optical potentials}

The optical potential is described as follows:

$$
\begin{aligned}
U= & \frac{2\left(E+m_{p} c^{2}\right)}{E+2 m_{p} c^{2}} \\
& \times\left[-V_{R} f_{R}+i\left\{4 W_{D} a_{D} \frac{d}{d r} f_{D}-W_{V} f_{V}\right\}+V_{C}\right] \\
& +\left(\frac{\hbar}{m_{\pi} c}\right)^{2}\left(V_{S O}+i W_{S O}\right) \frac{1}{r} \frac{d}{d r} f_{S o} l \dot{\sigma},
\end{aligned}
$$

where, $V_{R}, W_{D}, W_{V}, V_{S O}, W_{S O}, V_{C}$ stand for real volume, imaginary surface, imaginary volume, real and imaginary

\footnotetext{
${ }^{a}$ Presenting author, e-mail: shigyo@kune2a.nucl.kyushu-u.ac.jp
}

spin-orbit and Coulomb terms, respectively. These terms were originally suggested by Delaroche et al. [4] and modified by Kunieda et al. [5] for the intermediate mass range. In this study, the forms by Kunieda as following are used for Be and Li isotopes:

$$
\begin{aligned}
& V_{R}=\left\{V_{R 0}+V_{R 1} E^{\dagger}+V_{R 2} E^{\dagger 2}+V_{R 3} E^{\dagger 3}+V_{R 4} e^{-\lambda_{R} E^{\dagger}}\right\} \\
& \times\left[1+\frac{1}{V_{R 0}+V_{R 4}}(-1)^{Z_{p}+1} C_{V} \frac{N-Z}{A}\right] \\
& +C_{C} \frac{Z Z_{p}}{A^{1 / 3}} \phi_{C}\left(E^{\dagger}\right) \\
& W_{D}=\left\{W_{D 0}+(-1)^{Z_{p}+1} C_{W} \frac{N-Z}{A}\right\} \\
& \times e^{-\lambda_{D} E^{\dagger}} \frac{E^{\dagger 2}}{E^{\dagger 2}+W_{D 1}^{2}}, \\
& W_{V}=W_{V 0} \frac{E^{\dagger 2}}{E^{\dagger 2}+W_{V 1}^{2}}, \\
& V_{S O}=V_{S O 0} e^{-\lambda_{S O} E^{\dagger}} \text {, } \\
& W_{S O}=W_{S O 0} \frac{E^{\dagger 2}}{E^{\dagger 2}+W_{S O 1}^{2}}, \\
& \phi_{C}=\left(\lambda_{R} V_{R 4} e^{-\lambda_{R} E^{\dagger}}-V_{R 1}-2 V_{R 2} E^{\dagger}-3 V_{R 3} E^{\dagger 2}\right) \\
& \times\left[1+\frac{1}{V_{R 0}+V_{R 4}}(-1)^{Z_{p}+1} C_{V} \frac{N-Z}{A}\right],
\end{aligned}
$$

where, $V_{R 0}, V_{R 1}, V_{R 2}, V_{R 3}, V_{R 4}, W_{D 0}, W_{V 0}, V_{S O 0}$ and $W_{S O 0}$ are potential depths. The parameters $\lambda_{R}, \lambda_{D}, W_{D 1}, W_{V 1}, \lambda_{S O}$ express the energy variations of the potential depths. The variables $N, Z$ and $A$ correspond to nucleon, proton and neutron numbers in the target nucleus, respectively. The energy $E^{\dagger}$ is defined as $E-E_{f}$. The $E_{f}$ is the Fermi energy. The values $C_{V}, C_{C}, C_{W}$ denote coefficients and are fixed. The WoodsSaxon form is used for the nuclear radius and diffuseness, respectively.

$$
f_{i}(r)=\frac{1}{1+\exp \left\{\left(r-R_{i}\right) / a_{i}\right\}}, \quad i=R, D, V, S O,
$$

where, $R_{i}$ and $a_{i}$ are the geometry parameters. In this study, all $R_{i}$ and $a_{i}$ values are fixed. 

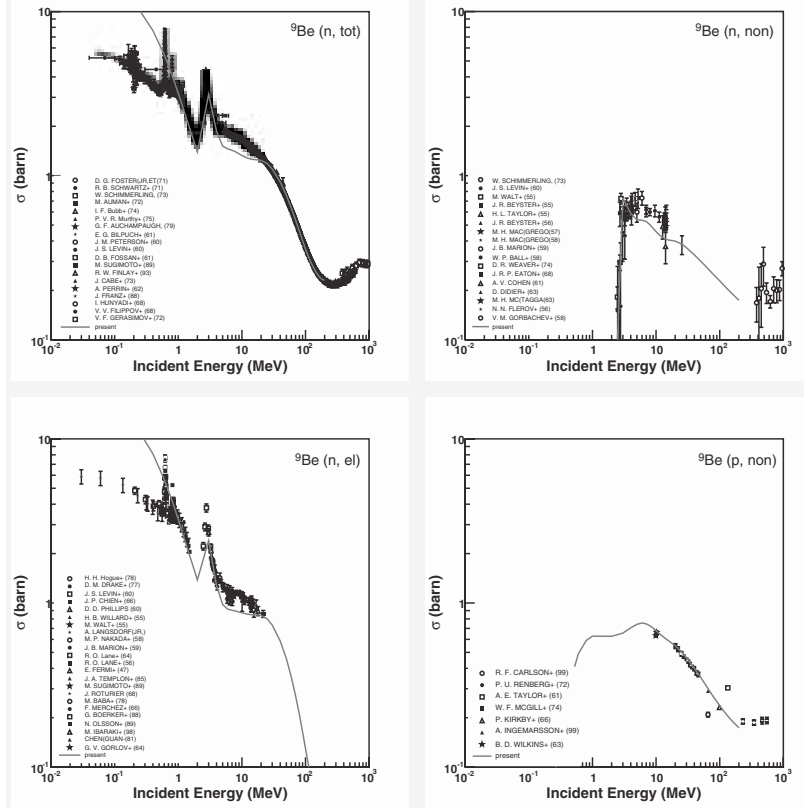

Fig. 1. The neutron incident total (upper left), nonelastic (upper right), elastic (bottom left) and proton incident nonelastic (bottom right) cross sections on ${ }^{9} \mathrm{Be}$.
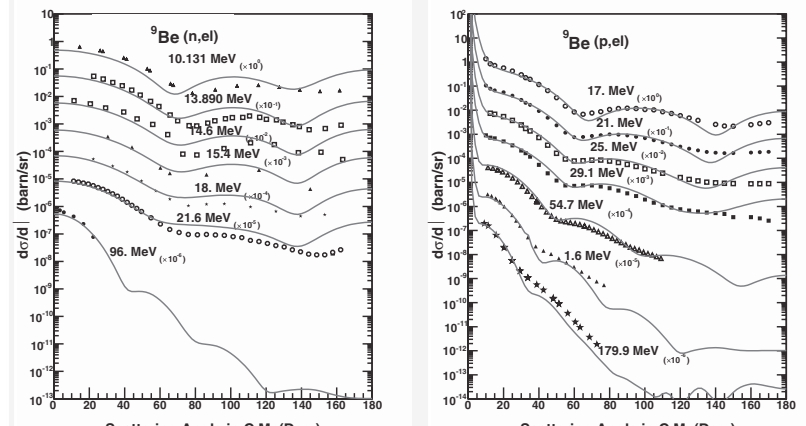

Fig. 2. The angular distributions of neutron (left pane) and proton (right pane) incident elastic scattering on ${ }^{9} \mathrm{Be}$.

Experimental data of cross sections are retrieved from the EXFOR data base [8]. The optimum parameters of the optical potential are searched for the $V_{R 0}, V_{R 1}, V_{R 2}, V_{R 3}$, $V_{R 4}, W_{D 0}, W_{V 0}, V_{S O 0}, W_{S O 0}, \lambda_{R}, \lambda_{D}, W_{D 1}, W_{V 1}, \lambda_{S O}$ by the ECIS-97 code[6] and the ROOT object-oriented data analysis framework [7]. At first, the parameters for ${ }^{9} \mathrm{Be}$ are searched because the number of experimental data for $\mathrm{Be}$ is larger than that for $\mathrm{Li}$ isotopes.

\section{Results}

Figure 1 shows neutron incident total [9-26] (upper left), nonelastic [11,17,27-41] (upper right), elastic cross sections $[17,19,27,33,42-60]$ (bottom left) and for proton incident nonelastic one [61-66] (bottom right) on ${ }^{9} \mathrm{Be}$. Lines and marks stand for the calculated values and experimental data,
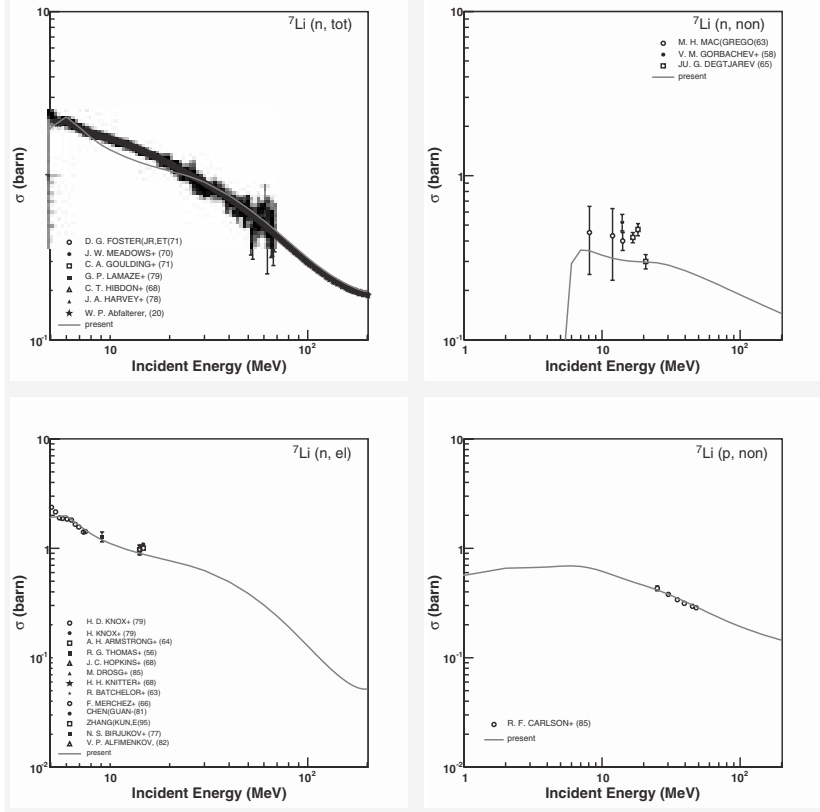

Fig. 3. The neutron incident total $[9,73-78]$ (upper left), nonelastic $[41,79,80]$ (upper right), elastic [55,59,81-90] (bottom left) and proton incident nonelastic [91] (bottom right) cross sections on ${ }^{7} \mathrm{Li}$.
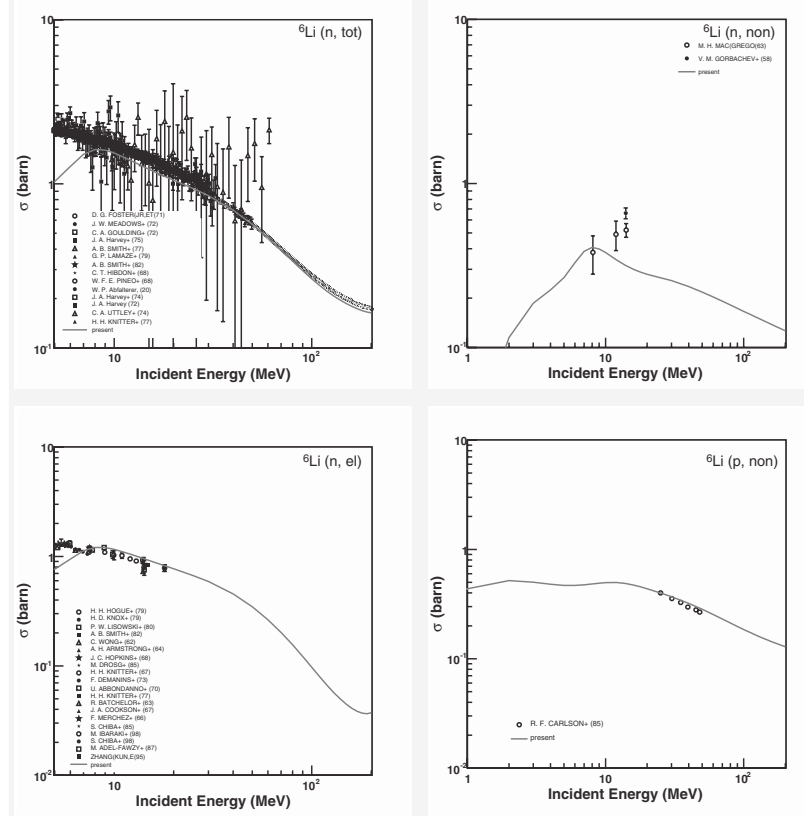

Fig. 4. The neutron incident total $[9,75,76,78,92-101]$ (upper left), nonelastic [41,79] (upper right), elastic [55,58,81,82, 84, 85, 87, 88, 96,101-111] (bottom left) and proton incident nonelastic [91] (bottom right) cross sections on ${ }^{6} \mathrm{Li}$.

respectively. One can see that the optical potentials obtained by the search reproduce experimental data above $20 \mathrm{MeV}$ for all type of cross sections. The calculated values particularly gives good agreement with the proton incident nonelastic scattering cross sections.

The angular distributions of elastic scattering on ${ }^{9} \mathrm{Be}$ are presented in figure 2. The left side is neutron incident data 

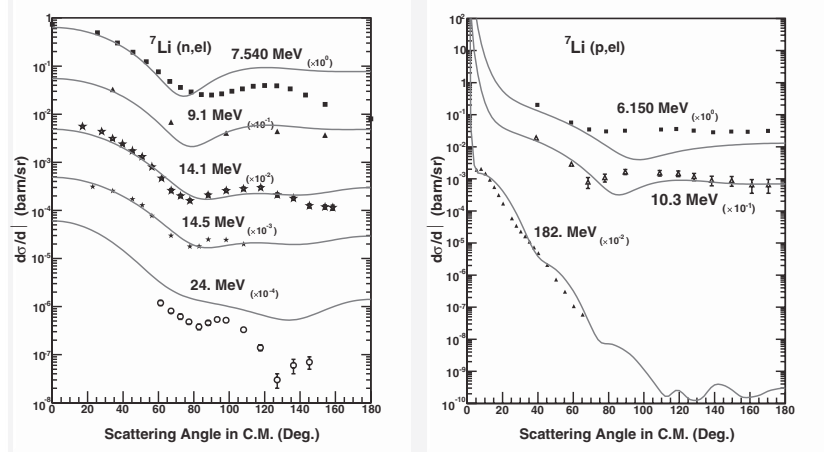

Fig. 5. The angular distributions of neutron $[55,87,89,112,113]$ (left pane) and proton $[114,115]$ (right pane) incident elastic scattering on ${ }^{7} \mathrm{Li}$.
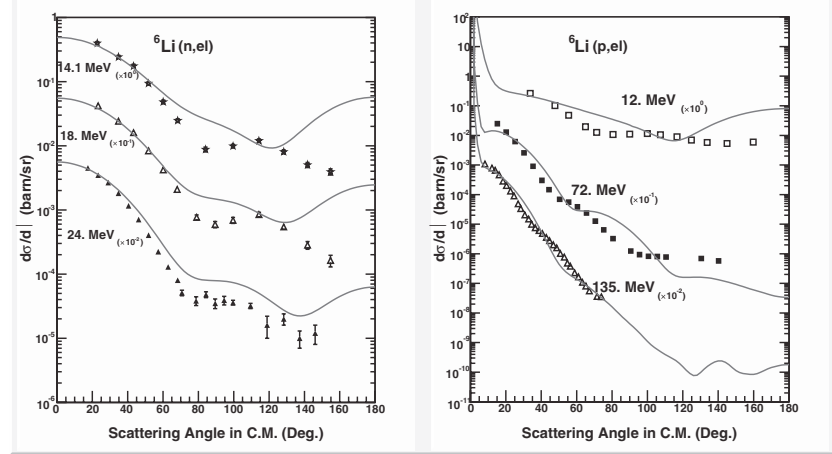

Fig. 6. The angular distributions of neutron $[58,110,113]$ (left pane) and proton [116-118] (right pane) incident elastic scattering on ${ }^{6} \mathrm{Li}$.

$[52,54,56-58,67,68]$ and the right side is proton ones [69$72]$, respectively. The calculations also reproduce the experimental data. Especially, the calculated values agree with the experimental ones for $96 \mathrm{MeV}$ neutron incident and 179.9 MeV proton incident elastic scattering.

Figures 3 and 4 stand for the neutron incident total, nonelastic, elastic cross sections and the proton incident nonelastic cross section for ${ }^{7} \mathrm{Li}$ and ${ }^{6} \mathrm{Li}$, respectively. In these figures, the calculated data give agreement with the experimental values. The calculation reproduce the neutron incident elastic scattering cross sections for ${ }^{7} \mathrm{Li}$ and proton incident nonelastic scattering ones for both $\mathrm{Li}$ isotopes.

The angular distributions of elastic scattering for ${ }^{7} \mathrm{Li}$ and ${ }^{6} \mathrm{Li}$ are presented in figures 5 and 6 . The left side is neutron incident data and the right side proton incident ones on each figure, respectively. The use of the obtained optical potential parameters are suitable for calculation of the angular distribution of elastic scattering cross section. In particular, the calculated values agree with the experimental ones of proton incident elastic scattering cross sections $182 \mathrm{MeV}$ incidence on ${ }^{7} \mathrm{Li}$ and $135 \mathrm{MeV}$ incidence on ${ }^{6} \mathrm{Li}$.

\section{Summary}

The parameters of optical potential for $\mathrm{Be}$ and $\mathrm{Li}$ were obtained in the energy region from 20 to $200 \mathrm{MeV}$. The neutron incident total, nonelastic, elastic, and the proton incident nonelastic cross sections by the calculation reproduce the experimental data above $20 \mathrm{MeV}$. These results will be introduced into JENDL-HE after the overall evaluation including particle emission cross sections.

Authors would like to thank the members of the Sigma Committee in Japan Atomic Energy Agency for useful suggestion.

\section{References}

1. K. Shibata et al., J. Nucl. Sci. Technol. 39, 1125 (2002).

2. Y. Watanabe et al., in Proceedings of International Conference on Nuclear Data for Science and Technology, 2004, edited by R.C. Haight, M. Chadwick, T. Kawano, P. Talou, AIP Conf. 769 (2005), p. 326.

3. P. Young et al., LAUR-88-382 (1988).

4. J.P. Delaroche et al., Phys. Rev. C 39, 391 (1989).

5. S. Kunieda et al., J. Nucl. Sci. Technol. (to be published).

6. J. Raynal, IAEA SMR-9/8, 281 (1970).

7. R. Brun, F. Rademakers, Nucl. Instrum. Meth. A 389, 81 (1997); See also the URL 〈http://root.cern.ch//.

8. Nuclear Data Centers Network, BNL-NCS-63330 (1996); See also the URL $\langle$ http://www.nea.fr/html/dbdata/x4/ $>$.

9. D.G. Foster, D.W. Glasgow, Phys. Rev. C 3, 576 (1971).

10. R.B. Schwartz et al., J. Bull. American Phys. Soc. 16, 495 (1971).

11. W. Schimmerling et al., Phys. Rev. C 7, 248 (1973).

12. M. Auman et al., Phys. Rev. 5, 1 (1972).

13. I.F. Bubb et al., Can. J. Phys. 52, 648 (1974).

14. G.F. Auchampaugh et al., Nucl. Sci. Eng. 69, 30 (1979).

15. E.G. Bilpuch et al., Washington AEC Office Reports 1034, 10 (1961).

16. J.M. Peterson et al., Phys. Rev. 120, 521 (1960).

17. J.S. Levin, L. Cranberg, Washington AEC Office Rep. 1029, 44 (1960).

18. D.B. Fossan et al., Phys. Rev. 123, 209 (1961).

19. M. Sugimoto et al., Nucl. Sci. Eng. 103, 37 (1989).

20. R.W. Finlay et al., Phys. Rev. C 47, 237 (1993).

21. J. Cabé, M. Cancé, Saclay Reports 4524 (1973).

22. A. Perrin et al., Comptes Rendus B 255, 277 (1962).

23. J. Franz et al., Nucl. Phys. A 490, 667 (1988).

24. I. Hyndai, I. Angeli, Nucl. Phys. 119, 525 (1968).

25. V.V. Filippov, M.N. Nikolaev, Nuclear Data for Computations Sem., Ass-68/17 (1968).

26. V.F. Gerasimov et al., Inst. Atomnoy Energii, Kurchatov Reports, 2222 (1972).

27. M. Walt, J.R. Beyster, Phys. Rev. 98, 677 (1955).

28. J.R. Beyster et al., Phys. Rev. 98, 1216 (1955).

29. H.L. Taylor et al., Phys. Rev. 100, 174 (1955).

30. J.R. Beyster et al., Phys. Rev. 104, 1319 (1956).

31. M.H. Mac Gregor et al., Phys. Rev. 108, 726 (1957).

32. M.H. Mac Gregor et al., Phys. Rev. 111, 1155 (1958).

33. J.B. Marion et al., Phys. Rev. 114, 1584 (1959).

34. W.P. Ball et al., Phys. Rev. 110, 1392 (1958).

35. D.R. Weaver, J. Walker, J. Phys. D 7, 1122 (1974).

36. J.R.P. Eaton, J. Walker, Proceedings of Nuclear Cross-Sections Technology Conference, Vol. 1 (1968), p. 169.

37. A.V. Cohen, J. Nucl. Energy 14, 180 (1961).

38. D. Didier, H. Dilleman, J. Phys. (France) 24, 85 (1963).

39. M.H. Mc-Taggart, H. Goodfellow (private communication). 
40. N.N. Flerov, V.M. Talysin, Atom. Energiya 1, 155 (1956).

41. V.M. Gorbachev, L.B. Poretskij, Atom. Energiya 2, 191 (1958).

42. H.H. Hogue, et al., Nucl. Sci. Eng. 68, 38 (1978).

43. D.M. Drake, et al., Nucl. Sci. Eng. 63, 401 (1977).

44. J.P. Chien, A.B. Smith, Nucl. Sci. Eng. 26, 500 (1966).

45. D.D. Phillips, Washington AEC Office Reports 1028 (1960), p. 29.

46. H.B. Willard et al., Phys. Rev. 98, 669 (1955).

47. A. Langsdorf et al., Phys. Rev. 107, 1077 (1957).

48. M.P. Nakada et al., Phys. Rev. 110, 1439 (1958).

49. R.O. Lane et al., Phys. Rev. B 133, 409 (1964).

50. R.O. Lane, J.E. Monahan, Argonne National Laboratory Report series 5554, 22 (1956).

51. E. Fermi, L. Marshall, Phys. Rev. 71, 666 (1947).

52. J.A. Templon et al., Nucl. Sci. Eng. 91, 451 (1985).

53. J. Roturier, Thesis (1968).

54. M. Baba et al., Proceedings of International Conference on Neutron Physics and Nuclear Data (1978), p. 198.

55. F. Merchez et al., Proceedings of Nuclear Data for Reactors Conference (1966), p. 393.

56. G. Boerker, et al., Proceedings of Conference on Nuclear Data for Science and Technology (1988), p. 193.

57. N. Olsson et al., Nucl. Phys. A 509, 161 (1990).

58. M. Ibaraki et al., J. Nucl. Sci. Technol. 35, 843 (1998).

59. Chen Guan-Ren et al., Chinese J. Nucl. Phys. 3, 320 (1981).

60. G.V. Gorlov et al., Doklady Akademii Nauk 158, 574 (1964).

61. P.U. Renberg et al., Nucl. Phys. A 183, 81 (1972).

62. A.E. Taylor, E. Wood, Nucl. Phys. 25, 642 (1961).

63. W.F. Mcgill et al., Phys. Rev. C 10, 2237 (1974).

64. P. Kirkby, W.T. Link, Can. J. Phys. 44, 1847 (1966).

65. A. Ingemarsson et al., Nucl. Phys. A 653, 341 (1999).

66. B.D. Wilkins, G. Igo, Phys. Rev. 129, 2198 (1963).

67. L.F. Hansen et al., Phys. Rev. C 31, 111 (1985).

68. G.L. Salmon, Nucl. Phys. 21, 15 (1960).

69. D.G. Montague et al., Nucl. Phys. A 199, 433 (1973).

70. L.J. De Bever et al., Nucl. Phys. A 579, 13 (1994).

71. H. Seifert, Thesis (1990).

72. S. Dixit et al., Phys. Rev. C 43, 1758 (1991).

73. J.W. Meadows, J.F. Whalen, Nucl. Sci. Eng. 41, 351 (1970).

74. C.A. Goulding et al., Chicago Operations Office, A.E.C., Contract rept. 3058-1, (1971), p. 11.

75. G.P. Lamaze et al., Proceedings of Conference on Cross Sections for Technology (1979), p. 48.

76. C.T. Hibdon, F.P. Mooring, Proceedings of Nuclear CrossSections Technology Conference, Vol. 1 (1968), p. 159.

77. J.A. Harvey et al., US DOE Nuclear Data Committee Reports 12 (1978), p. 229.

78. W.P. Abfalterer et al., Phys. Rev. C 63, 044608 (2001).

79. M.H. Mac Gregor et al., Phys. Rev. 130, 1471 (1963).

80. Ju.G. Degtjarev, Atom. Energiya 19, 456 (1965).
81. H.D. Knox et al., Nucl. Sci. Eng. 69, 223 (1979); H. Knox, R.O. Lane, Proceedings of Conference on Nuclear Cross Sections for Techonolgy (1979), p. 783.

82. A.H. Armstrong et al., Nucl. Phys. 52, 505 (1964).

83. R.G. Thomas et al., Phys. Rev. 101, 759 (1956).

84. J.C. Hopkins et al., Nucl. Phys. A 107, 139 (1968).

85. M. Drosg et al., Proceedings of Conference on Nuclear Data for Basic Science, Vol. 1 (1985), p. 145.

86. H.H. Knitter, M. Coppola, Proceedings of Nuclear CrossSections Technology Conference, Vol. 2 (1968), p. 827.

87. R. Batchelor, J.H. Towle, Nucl. Phys. 47, 385 (1963).

88. Zhang Kun et al., High Energy Phys. Nucl. Phys. 19, 879 (1995).

89. N.S. Birjukov et al., Proceedings of All Union Conference on Neutron Physics, Vol. 2 (1977), p. 27.

90. V.P. Alfimenkov et al., Yad. Fiz. 35, 542 (1982).

91. R.F. Carlson et al., Nucl. Phys. A 445, 57 (1985).

92. J.W. Meadows, J.F. Whalen, Nucl. Sci. Eng. 48, 221 (1972).

93. C.A. Goulding et al., Chicago Operations Office, A.E.C., Contract rept. 3058-21 (1972), p. 2.

94. J.A. Harvey, N.W. Hill, Proceedings of Conference on Nuclear Cross-Sections and Technology, Vol. 1 (1975), p. 244.

95. A.B. Smith et al., Argonne National Laboratory Reports (1977), p. 29.

96. A.B. Smith et al., Nucl. Phys. A 373, 305 (1982).

97. W.F.E. Pineo, J.A. Farrell, Proceedings of Nuclear CrossSections Technology Conference, Vol. 1 (1968), p. 153.

98. J.A. Harvey, N.W. Hill, Oak Ridge National Lab. Reports 4937 (1974), p. 187.

99. J.A. Harvey (private communication).

100. C.A. Uttley, K.M. Diment (private communication).

101. H.H. Knitter et al., Euratom Reports 5726 (1977), p. 1.

102. H.H. Hogue et al., Nucl. Sci. Eng. 69, 22 (1979).

103. P.W. Lisowski et al., Los Alamos Scientific Lab. Reports 8342 (1980).

104. C. Wong et al., Nucl. Phys. 33, 680 (1962).

105. H.H. Knitter, M. Coppola, Euratom Reports 3454 (1967).

106. F. Demanins et al., Inst. Naz. Fisica Nucleare Reports $73 / 2$ (1973).

107. U. Abbondanno et al., Nuovo Cimento A 66, 139 (1970).

108. J.A. Cookson et al., Nucl. Phys. A 91, 273 (1967).

109. S. Chiba et al., J. Nucl. Sci. Technol. 22, 771 (1985).

110. S. Chiba et al., Phys. Rev. C 58, 2205 (1998).

111. M. Adel-Fawzy et al., Nucl. Instrum. Meth. 169, 533 (1980).

112. R.J. Danahy, Thesis (1980).

113. L.F. Hansen et al., Phys. Rev. C 38, 525 (1988).

114. K. Kilian et al., Nucl. Phys. A 126, 529 (1969).

115. A. Johansson et al., Arkiv foer Fysik 19, 541 (1961).

116. W.D. Harrison, A.B. Whirehead, Phys. Rev. 132, 2607 (1963).

117. R. Henneck et al., Nucl. Phys. A 571, 541 (1994).

118. R.S. Henderson et al., Nucl. Phys. A 372, 117 (1981). 